\title{
NRGI types down regulate in methamphetamine and heroin dependent patients
}

\begin{abstract}
Methamphetamine is a highly addictive neurotoxin stimulant. Its neurotoxic effects may cause brain damage and deficiencies in several central nervous system functions. Heroin is a highly addictive opioid drug with euphoric effects and acute and chronic influence in mood and behavior. Schizophrenia (SCZ) is a major complex psychiatric disorder with no clarified etiology or biological diagnosis. Neuregulin1 is one of four proteins in the neuregulin family that act on the EGFR family of receptors. NRG1 gene (8p21.1-22), is essential for the normal development of the nervous system and the heart. At least six major types of NRG1 exist in humans. Types I (acetylcholine receptor activator), II (glial growth Factor) and III (sensory and motor neuron-derived factor) are expressed in excitatory and inhibitory neurons, as well as astrocytes, and neuronal activity. NRG1 was indicated as marker gene for schizophrenia and may changes in substances abuse induced psychosis.

Present study was assessed expression of types I, II and III of NRG1 gene in total blood samples of 150 Meth-dependence, 150 Heroin dependent, $150 \mathrm{SCZ}$ patients and 150 non psychiatric subjects by using quantitative Real time PCR along with PANSS and Wechsler III clinical tests.

Findings were showed significant $(\mathrm{p}<0.05)$ down regulation of all three types of NRG1 genes, in Meth dependent and SCZ patients in compare with non psychiatric subjects. Down regulation of type III in Heroin abusers in compare with non psychiatric subjects has been detected. Also significant down regulation of types I and III in Methdependents in compare with SCZ patients were detected. Several correlations were found between mRNA levels and psychiatric tests results.

Results were presented NRG1 as a potential peripheral marker for Meth-dependence as well as schizophrenia that could explain similar symptoms of both disorders. In addition down expression of type I and type III in Meth-dependence in compare with SCZ group may relate to chronic effects of methamphetamine abuse.
\end{abstract}

Keywords: NRG1, schizophrenia, meth dependent, heroin dependent, gene expression
Volume 3 Issue 2 - 2017

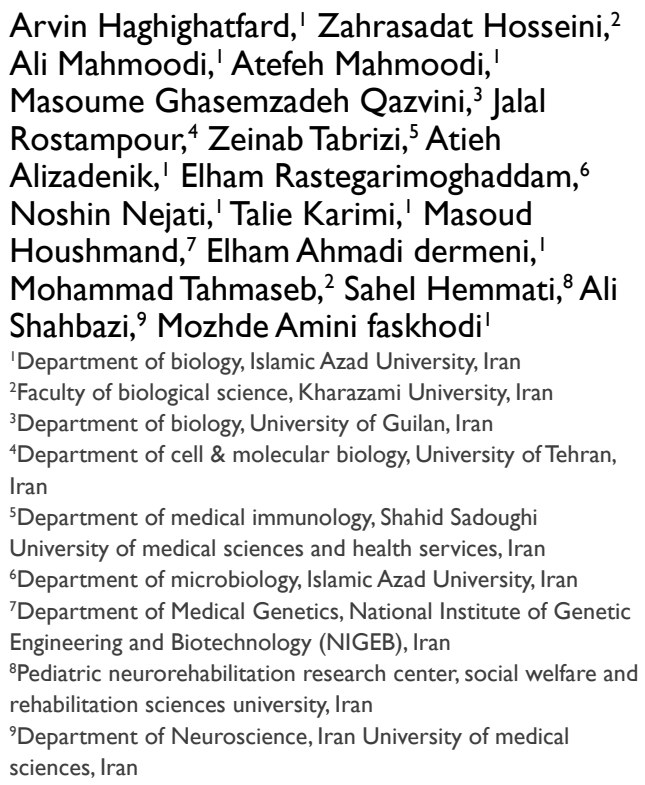

Correspondence: Ali Shahbazi, Department of Neuroscience, Iran University of medical sciences, Tehran, Iran, Tel 9809355 I27310,Email arvinland@yahoo.com

Received: September 25, 2016 | Published: March 22, 2017
Abbreviations: CNS, central nervous system; NRG1, neuregulin 1; EGFR, estimated glomerular filtration rate; DSM-V, diagnostic and statistical manual of mental disorders; PANSS, positive and negative syndrome scale; WAIS-IV, wechsler adult intelligence scale; PGK1, phosphoglycerate kinase 1

\section{Introduction}

Heroin is a highly addictive opioid pain killer which was used as a recreational drug for its euphoric effects. ${ }^{1}$ Methamphetamine is a strong central nervous system (CNS) stimulant which is mainly used as a recreational drug and used for treatment of attention deficit hyperactivity disorder and obesity until the 70th decade of 20th centuries. ${ }^{2}$ Methamphetamine may cause a highly psychological dependence along with structural and functional abnormalities in brain. Psychotic symptoms in chronic abusers of methamphetamine and less frequently in chronic abusers of Heroin are previously reported. Recent estimates were indicated about $40 \%$ of Methamphetamine abusers affected with psychosis. Almost all of psychotic symptoms of substance abusers are commonly presenting on patients with schizophrenia. $^{3}$

Schizophrenia is one of the most common and well known psychiatric disorders which developed in adolescents and young adults with $1 \%$ prevalence in population worldwide. Schizophrenia is characterized by positive symptoms (such as hallucinations, delusions and paranoia), negative symptoms (such as flat or blunted affect, poor speech, and social withdrawal), and cognitive deficits (such as memory deficits, decrease on attention, and impairment in executive functions). ${ }^{4}$ Molecular mechanisms of psychotic symptoms in substance abusers and schizophrenia are not clarified. By the way involvement of several genes in etiology of schizophrenia and severity of patient's symptoms had been reported. NRG1 (Neuregulin 1) is a candidate gene in schizophrenia which is located in $8 p 12$ with more than 21 exons and tremendous number of isoforms that probably is due to multiple promoters and alternative splicing. These isoform could be grouped in six types based on their structures and functions. Overall, NRG1 is one of four proteins in the neuregulin family that act on the EGFR family of receptors and as a growth factor plays roles in modulation of neuronal migration, synaptogenesis, gliogenesis, dendritic growth, neuron-glia communication, myelination, and neurotransmission..$^{5}$ Type I or Heregulin is an acetylcholine receptor activator, type II is a Glial Growth Factor, type III is a sensory and motor neuron-derived factor and type IV is involved in neuronal activity regulation. Functions of types V and VI are not known. ${ }^{6}$ Types 
I, II and III of NRG1 express in human peripheral blood in addition to neurons, and types IV and V expressing specifically in brain. ${ }^{6}$

Association of several polymorphisms in NRG1 with schizophrenia, bipolar disorder and depression were reported $;^{7}$ but direction of NRG1 expression changes in schizophrenia and other behavioral characterizations are controversial. ${ }^{8}$ Law et al. ${ }^{9}$ were reported over expression of type I and Nicodemus et al. ${ }^{9}$ were reported down expression of type III both in hippocampus of schizophrenic patients. ${ }^{9}$ Zhang et al. ${ }^{10}$ have revealed down regulation of NRG1 mRNA level in peripheral blood of schizophrenia and Shibuya et al later were showed serum levels of NRG1 are decreased in patients with chronic schizophrenia. ${ }^{10}$

Present study aimed to assess mRNA level of types I, II and III of NRG1 in third group of patients, methamphetamine dependent, Heroin dependent, schizophrenic patients and one non-psychiatric control group with no reputation of drug abuse. Expression study was combined with psychiatric and psychological tests to investigate effects of substance abuse on NRG1 mRNA level and correlation of NRG1 expression with clinical situation and behavioral characteristics of chronic substance abusers.

\section{Material and methods}

\section{Subject selection}

Present study was included 150 non psychiatric subjects (95 male, 55 female) aged 30 to 56 years old, 150 schizophrenic patients (110 paranoid, 26 undifferentiated, 8 residual and 6 catatonic) (90 male, 60 female) aged 34 to 54 years old, 150 methamphetamine dependent subjects ( 84 male, 66 female) aged 28 to 52 years old and 150 Heroin dependent subjects (103 male, 47 female) aged 28 to 52 years old. All patients were diagnosed by two independent senior psychiatrists based on extended clinical interview and patients' chart review of DSM-V. Schizophrenic patients were started treatment, including antipsychotic medications in 17 (or more) month before sampling and were considered as chronic patients. Patients with schizoaffecive or schizotypal illness were excluded. Methamphetamine and Heroin abusers were pure abusers and dependent to substance at least for 12 months. Non psychiatric group was matched to patient's group for gender, age, race, socioeconomic situation, familial situation and education. None of the subjects had current or history of sever medical condition, neurological disorder, history of head trauma with loss of consciousness. Schizophrenic and non psychiatric subjects had no reputation of psycho-stimulant or opioid drug abuse and alcohol or nicotine dependence. Smoking tobacco and alcohol dependence is common in Schizophrenic patients and it may effects on gene expression results. To prevent these effects, all subjects were examined for serum (Microplate immunoassay kits, OraSure Technologies) and urine (AU 5822 analyzer, Beckman Coulter) cotinine for smoking abstinent. The cut point for cotinine positive in serum was \& gt; $0.457 \mathrm{ng} / \mathrm{mL}$ and for cotinine in urine was \& gt; 500 ng/mL. Cell Biolabs' Alcohol Assay Kit (Cat No: STA-620) had been used for alcohol abstinent testing. All subjects had informed consent and filled a written consent form in Persian which was provided based on Helsinki declaration guidelines. Study has been approved by local ethical committee.

\section{PANSS}

Symptom severity was measured with the Positive and Negative Syndrome Scale (PANSS), is a 30 -item semi-structured interview that was designed to evaluate three major symptom categories associated with schizophrenia: positive, negative and general symptoms. ${ }^{11}$

\section{Wechsler adult intelligence scale (WAIS-IV)}

The Wechsler Adult Intelligence Scale (WAIS) is a test designed to measure intelligence in adults and older adolescents. Forward and backward digit span are two subtests of WAIS which calculate the subject's short-term memory or working memory capacity. ${ }^{12}$

\section{Blood sampling and real time PCR}

Gene expression evaluation was performed based on previous related studies. Briefly, blood $(5 \mathrm{ml})$ was collected from the cubital vein without tourniquet by using PAXgene blood RNA tubes (Cat No762165) between 10.00 and 11.00 AM. Total RNA was extracted from peripheral blood samples according to standard protocols using by RNA Purification kit (GeneJETTM RNA Purification Kit\#K0732, Fermentas, Latvia). Quality of extracted RNA checked by gel electrophoresis with $1 \%$ Agarose. The cDNA was synthesized using a Transcription First Strand cDNA Synthesis Kit (RevertAid Premium First Strand cDNA Synthesis Kit \#K1652, Fermentas, Latvia) according to manufacturer's protocol. Primers for all genes designed by "oligo7" software and blasted on NCBI website. PGK1 were used for normalization as endogenous reference gene. Table 1 presented primer sequences. Standard curves for each gene were prepared using serial dilutions (1:4) of pooled cDNA from total extracted RNA of five non-psychiatric subjects. In each experiment, the R2 value of the standard curve was more than 0.99 and no-template control assays resulted in no detectable signal. Quantitative RT-PCR was performed by using SYBR green (Thermo Scientific Maxima SYBR Green/ROX qPCR Master Mix (2X) \#K0221, Fermentas, Latvia). Quantitative RT-PCR was performed in triplicate method by using CFX96 Touch Real-Time PCR Detection System (BIO-RAD, California, United States). In qPCR tests outliers or failures samples repeated for each gene. Ratio calculated by using pfafle formula.

Table I Primers of genes

\begin{tabular}{ll}
\hline Genes primer & primer sequence \\
\hline PGKI forward primer & 5'GAGCCGACTTGTTCTCTCGT3' \\
PGKI reverse primer & 5'GCGGCTAGAAACCACCAAAC3' \\
NRGI type I forward primer & 5'AGCCTCAACTGAAGGAGCAT3' \\
NRGI type I reverse primer & 5'ACTCCCCTCCATTCACACAG3' \\
NRGI type II forward primer & 5'CCCTATCTGGTGAAGGTGCA3' \\
NRGI type II reverse primer & 5'GTCGGGCTCCATGAAGAAGA3' \\
NRGI type III forward primer & 5'ATCTGCATTGTCCCCATCCT3' \\
NRGI type III reverse primer & 5'GCAGTTGCGTCCAGAGAAAT3' \\
\hline
\end{tabular}

\section{Statistical tests}

Descriptive data are expressed as mean $\pm \mathrm{SD}$ (range) and level of statistical significance was set at $\mathrm{P}<0.05$.Compliance with normal distribution for continuous variables was assessed via the Kolmogorov-Smirnov test. Statistical differences were calculated by one-way ANOVA followed by independent Student's $t$ test in multiple group comparisons. Pearson correlation analysis was conducted to determine the relationship between variables. Bonferroni correction 
was used for multiple comparisons corrections. Statistical analyses were conducted by using SPSS version 23 .

\section{Results}

Clinical and demographic details have been presented in Table 2.

Table 2 Demographic and clinical data

\begin{tabular}{|c|c|c|c|c|}
\hline Variables & SCZ & Meth abusers & Heroin abusers & $\begin{array}{l}\text { Non psychiatric } \\
\text { subjects }\end{array}$ \\
\hline Gender & 90 male, 60 female & 84 male, 66 female & I03 male, 47 female & 95 male , 55 female \\
\hline Age & $38 \pm 5.9$ & $36 \pm 7$ & $35 \pm 5.3$ & $33 \pm 7.6$ \\
\hline Age of onset(years) & $21.5 \pm 8.5$ & - & - & - \\
\hline Duration of illness(month) & $8.4 \pm 15.6$ & - & - & - \\
\hline $\begin{array}{l}\text { Duration of } \\
\text { hospitalization(day) }\end{array}$ & $19.13 \pm 5.3$ & - & - & - \\
\hline \multirow[t]{2}{*}{ Cotinine level (ng/mL) } & $\begin{array}{l}\text { Serum level: } 0.94 \pm 0.26 \\
\mathrm{ng} / \mathrm{mL}\end{array}$ & $\begin{array}{l}\text { Serum level: } \\
0.87 \pm 0.23 \mathrm{ng} / \mathrm{mL}\end{array}$ & $\begin{array}{l}\text { Serum level: } \\
0.134 \pm 0.09 \mathrm{ng} / \mathrm{mL}\end{array}$ & $\begin{array}{l}\text { Serum level: } 0.33 \pm 0.2 \\
\mathrm{ng} / \mathrm{mL}\end{array}$ \\
\hline & $\begin{array}{l}\text { urine level: } 125 \pm 0.27 \mathrm{ng} / \\
\mathrm{mL}\end{array}$ & $\begin{array}{l}\text { urine level: II } 3 \pm 0.14 \\
\mathrm{ng} / \mathrm{mL}\end{array}$ & $\begin{array}{l}\text { urine level: I77 } \pm 0.08 \mathrm{ng} / \\
\mathrm{mL}\end{array}$ & $\begin{array}{l}\text { urine level: } 42 \pm 0.17 \\
\mathrm{ng} / \mathrm{mL}\end{array}$ \\
\hline \multirow[t]{5}{*}{$\begin{array}{l}\text { Antipsychotic } \\
\text { medications(mg/day) }\end{array}$} & Clozapine: 600 & Risperidone: 4 & Risperidone: 4 & - \\
\hline & Clonazepam: 0.5 & & & \\
\hline & Risperidone: 2 & & & \\
\hline & Olanzapine: 20 & & & \\
\hline & Fluoxetine: 50 & & & \\
\hline PANSS total & $73.25 \pm 17.66$ & $61.29 \pm 12.33$ & $53.25 \pm 24.43$ & - \\
\hline General symptoms & $28.4 \pm 6.92$ & $21.3 \pm 6.55$ & $19.4 \pm 5.33$ & - \\
\hline Positive score & $23.49 \pm 8.43$ & $29.61 \pm 7.16$ & $16.98 \pm 9.72$ & - \\
\hline Negative score & $19.54 \pm 8.38$ & $19.77 \pm 9.11$ & $17.88 \pm 6.49$ & - \\
\hline IQ total score & $84.43 \pm 11.7$ & $86.23 \pm 14.2$ & $91.35 \pm 18.5$ & $96.33 \pm 5.23$ \\
\hline Forward digit span & $4.82 \pm 1.13$ & $3.22 \pm 1.36$ & $5.29 \pm 1.80$ & $6.84 \pm 1.52$ \\
\hline Backward digit span & $1.88 \pm 1.29$ & $1.43 \pm 2.30$ & $2.65 \pm 1.61$ & $3.73 \pm 1.76$ \\
\hline
\end{tabular}

SCZ, Schizophrenia; Meth, Methamphetamine; PANSS, positive and negative symptom scale; IQ, intelligence quality

\section{mRNA levels results}

Significant down expression of mRNA levels of type I $(\mathrm{P}=0.001)$, type II $(\mathrm{P}=0.001)$ and type III $(\mathrm{P}=0.001)$ were detected in peripheral blood of schizophrenic patients in compare with non psychiatric subjects. Significant down regulation of mRNA levels of type I $(\mathrm{P}=0.001)$, type II $(\mathrm{P}=0.001)$ and type III $(\mathrm{P}=0.001)$ have been detected in peripheral blood of methamphetamine abusers in compare with non psychiatric subjects. In Heroin abusers vs. non psychiatric subjects significant down regulation in mRNA levels of type III $(\mathrm{P}=0.001)$ was revealed. mRNA level of type I $(\mathrm{P}=0.003)$ and type III $(\mathrm{P}=0.002)$ were significantly decreased in Methamphetamine abusers in compare with schizophrenic patients and Heroin abusers. $p$ values and ratio for every types in each comparison have presented in Table 3.

\section{PANSS results and correlations with gene expressions}

Significant correlations were found between total score, positive symptoms score and negative symptoms score of PANSS with gene expression results. Significant correlations were found between increase in PANSS positive and negative score with down regulation of type I (positive symptoms $p$ value $=0.002$ and $R$ value $=0.76$, negative symptoms $\mathrm{p}$ value $=0.004$ and $\mathrm{R}$ value $=0.64$ ) and type III (positive symptoms $\mathrm{p}$ value $=0.001$ and $\mathrm{R}$ value $=0.68$, negative symptoms $\mathrm{p}$ value $=0.002$ and $R$ value $=0.57$ ) in schizophrenic patients and type I (positive symptoms $\mathrm{p}$ value $=0.002$ and $\mathrm{R}$ value $=0.71$, negative symptoms $\mathrm{p}$ value $=0.002$ and $\mathrm{R}$ value $=0.59$ ) and type III (positive symptoms $\mathrm{p}$ value $=0.001$ and $\mathrm{R}$ value $=0.68$, negative symptoms $\mathrm{p}$ value $=0.001$ and $R$ value $=0.77$ ) in Methamphetamine abusers.

\section{WAIS-IV results and correlation with gene expressions}

There was no significant difference between groups in IQ total score but forward and backward digit span results were showed significant deficit in working memory in schizophrenic patients and Methamphetamine abusers. No significant correlation was found between IQ total score and gene expression in patients but decrease in digit span (forward and backward) results were significantly 
correlated with down regulation of type III in both schizophrenic (p value $=0.005$ ) patients and Methamphetamine abusers $(p$ value $=0.003)$.
No significant correlation was found between demographic data of patients and gene expression results.

Table 3 Gene expression ratio and p value for expression of NRGI types between groups

\begin{tabular}{|c|c|c|c|c|c|c|}
\hline Gene & $\begin{array}{l}\text { SCZ vs. } \\
\text { Normal }\end{array}$ & $\begin{array}{l}\text { Meth vs. } \\
\text { Normal }\end{array}$ & $\begin{array}{l}\text { Heroin vs. } \\
\text { Normal }\end{array}$ & $\begin{array}{l}\text { Meth vs. } \\
\text { SCZ }\end{array}$ & $\begin{array}{l}\text { Meth vs. } \\
\text { Heroin }\end{array}$ & $\begin{array}{l}\text { Heroin vs. } \\
\text { SCZ }\end{array}$ \\
\hline \multirow[t]{2}{*}{ NRGI type I } & Ratio: 0.64 & Ratio: 0.42 & Ratio: 0.95 & Ratio: 0.77 & Ratio: 0.45 & Ratio: I.34 \\
\hline & $P$ value: 0.001 & $P$ value: 0.001 & $P$ value: 0.32 & $P$ value: 0.003 & $P$ value: 0.001 & $P$ value: $0.00 \mathrm{I}$ \\
\hline \multirow[t]{2}{*}{ NRG I type II } & Ratio: 0.53 & Ratio: 0.6 & Ratio: 0.87 & Ratio: 0.89 & Ratio: 0.57 & Ratio: I.19 \\
\hline & $P$ value: 0.001 & $P$ value: 0.001 & $P$ value: 0.09 & $P$ value: 0.14 & $P$ value: 0.001 & $P$ value: 0.04 \\
\hline \multirow[t]{2}{*}{ NRG I type III } & Ratio: 0.66 & Ratio: 0.4 & Ratio: 0.64 & Ratio: 0.72 & Ratio: 0.68 & Ratio: 0.83 \\
\hline & $P$ value: $0.00 \mathrm{I}$ & $P$ value: 0.001 & $P$ value: 0.001 & $P$ value: 0.002 & $\mathrm{P}$ value: 0.07 & $P$ value: 0.04 \\
\hline
\end{tabular}

\section{SCZ, Schizophrenia; Meth, Methamphetamine}

\section{Discussion}

Present study confirms NRG1 involvement in schizophrenia and presents NRG1 as biomarker for schizophrenia. Gene expression results have confirmed Shibuya et al results about decrease of serum levels of NRG1 in peripheral blood of schizophrenic patients. ${ }^{5}$ Similar down regulation in all three types, in chronic methamphetamine abusers were detected which could be related to sever and constant neurodegenration of CNS. Majority of NRG1 types (I, II, and III) are expressed in excitatory neurons, and also present in GABAergic neurons and astrocytes. Neural activity regulates expression of NRG1 isoforms, and leads to significant increase in level of type I and IV NRG1proteins. ${ }^{7}$ So these down expression could be related to low neural activity in methamphetamine and Heroin abusers as well as schizophrenic patients. In addition NRG1 are involved in several CNS functions including neuronal migration, synaptogenesis, dendritic growth, neuron-glia communication, myelination, and neurotransmission which are necessary to connectivity and executive functions such as memory, and attention. Deficiency of memory in schizophrenic patients and methamphetamine abusers which were correlated with type 3 down regulation may supports reports about NRG1 down expression in hippocampus as main region of memory in schizophrenic patients and also provide evidence about shared affected mechanisms between schizophrenia and methamphetamine dependence. ${ }^{6-9}$

Correlations of NRG1 expression with psychiatric tests bring important findings about NRG1 role in behavior and substance induced psychosis. Types I and III down expression were significantly associated with severity of positive symptoms such as paranoia and negative symptoms such as withdrawal and Retardation. It seems that down expression of NRG1 cause lack of functions in excitatory neurons that leads to lack of emotional activity in subjects as well as deficiency in reasonable thinking that cause paranoid thoughts. In the other hand same results in correlation studies between schizophrenia and Methamphetamine abusers could explain the high frequency of Meth-psychosis with schizophrenic like symptoms among the Methamphetamine abusers. ${ }^{2}$

Prevalence of smoking in schizophrenic patients is higher than general population as well as patients with other psychiatric conditions. In the other hand while neuregulin signaling pathway were indicated as involved pathway in etiology of schizophrenia, genes from the same pathway such as NRG3 were found associated with smoking behavior tendency. ${ }^{13}$ These shared genetic bases, could be related to impulsivity mechanisms which have been affected in several psychiatric disorders such as ADHD and schizophrenia. Finding may present NRG1 as new shared candidate gene between schizophrenia and addictive behaviors especially in stimulants. Down regulation of NRG1 types in SCZ and addict subjects compared with non-psychiatrics, were similar. It's possible that the same genetic or epigenetic mechanism which leads down expression of NRG1 in SCZ, down regulates NRG1 in methamphetamine and Heroin abusers even before drug abuse. It may explain the tendency of schizophrenic patients to opiuid and stimulants abuse. However it seems that chronic abuse of these addictive substances will decrease the NRG1 mRNA level more that may be involved in drug induced psychosis.

\section{Limitation}

Uncertainty of gene expression study in peripheral blood in neuropsychiatric patients is always an obstacle.

\section{Conclusion}

Results were confirmed that expression level of NRG1 changes in peripheral blood of schizophrenic patients. In addition same direction but more sever expression alteration of all three NRG1 types were detected in Methamphetamine dependents in compare with schizophrenics that may explain similar symptoms of Methamphetamine induced psychosis with schizophrenia. In Heroin dependents, NRG1 Type III was showed similar expression alteration with Methamphetamine dependents and schizophrenic patients. Study present NRG1 (especially type III) as potential peripheral marker for prediction of substance abuse psychosis. Also study have report that NRG1 down expression effects (especially type III) on working memory functions and severity of negative symptoms in schizophrenic patients and Methamphetamine dependents. Further studies with more comprehensive clinical and neuroimaging assessments with greater sample size could uncover the important role of NRG1 in schizophrenia and substance induced psychosis as well as behaviors and personality types. Also it may lead to development of new targeted therapeutic antipsychotics for schizophrenia and drug induced psychosis.

\section{Acknowledgements}

We would like to thank to young researchers and elites club of Islamic Azad University and its presidency Prof. Karim Zaree for all of their official and financial supports 


\section{Conflict of interest}

The author declares no conflict of interest.

\section{References}

1. Rook EJ, van Ree JM, van den Brink W, et al. Pharmacokinetics and pharmacodynamics of high doses of pharmaceutically prepared heroin, by intravenous or by inhalation route in opioid-dependent patients. Basic Clin Pharmacol Toxicol. 2006;98(1):86-96.

2. Glasner-Edwards S, Mooney LJ. Methamphetamine psychosis: epidemiology and management. CNS drugs. 2014;28(12):1115-1126.

3. McKetin R, Lubman DI, Baker AL, et al. Dose-related psychotic symptoms in chronic methamphetamine users: evidence from a prospective longitudinal study. JAMA psychiatry. 2013;70(3):319-324.

4. Deng C, Huang XF. Increased density of GABAA receptors in the superior temporal gyrus in schizophrenia. Exp Brain Res. 2006;168(4):587-590.

5. Harrison PJ, Law AJ. Neuregulin 1 and schizophrenia: genetics, gene expression, and neurobiology. Biol Psychiatry. 2006;60(2):132-140.

6. Liu X, Bates R, Yin DM, et al. Specific regulation of NRG1 isoform expression by neuronal activity. J Neurosci. 2011;31(23):8491-8501.

7. Bousman CA, Potiriadis M, Everall IP, et al. Effects of neuregulin-1 genetic variation and depression symptom severity on longitudinal patterns of psychotic symptoms in primary care attendees. Am J Med Genet B Neuropsychiatr Genet. 2014;165B(1):62-67.
8. Kukshal P, Bhatia T, Bhagwat AM, et al. Association study of neuregulin-1 gene polymorphisms in a North Indian schizophrenia sample. Schizophr Res. 2013;144(1-3):24-30.

9. Nicodemus KK, Law AJ, Luna A, et al. A 5' promoter region SNP in NRG1 is associated with schizophrenia risk and type III isoform expression. Mol Psychiatry. 2009;14(8):741-743.

10. Shibuya M, Komi E, Wang R, et al. Measurement and comparison of serum neuregulin 1 immunoreactivity in control subjects and patients with schizophrenia: an influence of its genetic polymorphism. J Neural Transm (Vienna). 2010;117(7):887-895.

11. Association AP. Diagnostic and statistical manual of mental disorders (DSM) DC. American psychiatric association, Washington, USA, 1994. p. 143-147.

12. Alan S kaufman, Elizabeth $\mathrm{O}$ Lichtenberger. Assessing adolescent and adult intelligence. USA: John Wiley \& Sons; 2005. 816 p.

13. Miranda L Fisher, Anu Loukola, Jaakko Kaprio, et al. Chapter Four-Role of the Neuregulin Signaling Pathway in Nicotine Dependence and Comorbid Disorders. International review of neurobiology. 2015;124:113131. 